\title{
61. Study of Vertebrobasilar Insuffisiency
}

\author{
Shinichiro Endo, Yoshio Sakuta, Kenshichiro Endo, Ichiro Ogawa, \\ Yaichi AKIBA, Hiroki IgURA, Tuneharu Higa and Hiroshi Suda \\ 2nd Dept. of Surgery, Fukushima Medical College
}

Twenty-six patients of basilar arterial insufficiency encountered in our clinic were reported with special emphasis on some diagnostic problems. The manifested clinical pictures of these cases, ranging from 15 to 64 in age, were found to be headache, vertigo, tinnitus, ataxic gait, impaired equilibration and other less frequent symptoms (nausea, vomitting, visual disturbance).

Serial Vertebral Angiography revealed the following findings:

1. non- or poor-filling of the basilar branches in 7 cases.

2. non- or poor-filling of the basilar stem in 9 cases.

3. non-filling of the vertebral artery in 4 cases.

4. markedly tortuous basilarstem in 2 cases. Vertebral in 4 cases.

3 cases of homonymous hemianopsia have a non or poor visualized posterior cerebral artery.

These cases of vertebrobasilar insufficiency returned to fair filling in parallel with improvement of clinical manifestation under the treatment of the systemic administration of vasodilator, sympathetic inhibiting agens and blocking of the stellate ganglion.

\section{Vertebral Artery Insufficiency Caused by Cervical Spodylosis}

\section{-With Special Reference with a New Surgical Technique-}

\author{
Chikao NAGASHIMA
}

Dept. of Neurosurgery, Moro General Hospital, Saitama-ken

A new surgical technique of vertebro-basilar insufficiency due to narrowing and kinking of the vertebral artery by cervical spondylosis was presented with $16 \mathrm{~mm}$. movie picture. The results obtained from 17 cases treated on this method with longest 24 months follow-up have been sufficiently gratifying to justify a description of the operation.

1) The uncal osteophyte, fibro-cartilaginous mass and part of uncal region of vertebral body which compressing the artery are removed with an electric drill or Hall drill through the anterior approach. Sectioning of the longus colli muscle 
greatly facilitate to obtain full exposure of the transverse process and uncal osteophyte. No interbody fusion required. (Fig. 1-A and B)

2) Unrofing of the foramen transversarium of two levels above and below the site where the artery is potentially kinked. (Fig. 1-B and C)

3) The vertebral artery was decompressed from its compromised bony structures, although a crease, remnant of periosteum and fibrosis remained on its adventitia with constriction and less pulsatile at the site. Procaine solution is injected immediately beneath the adventitia. While the adventitia is balooned from the media by procaine solution, a dural hook is inserted into the adventitia and incision is made. Removal of anterior one half of the perivascular fibrosis with adventitia and denervation of the vertebral artery are accomplished. (Fig. 2-D and $\mathrm{E}$ )

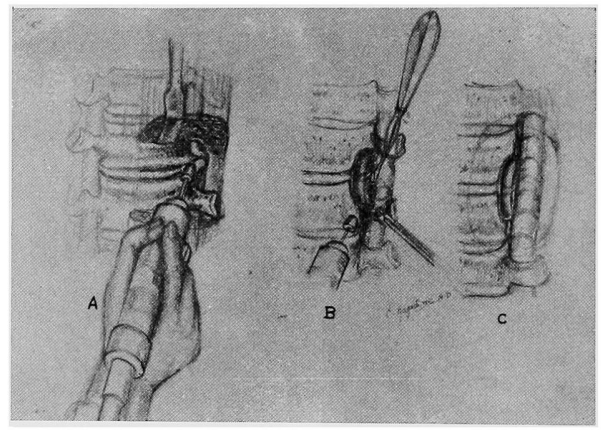

Fig. 1

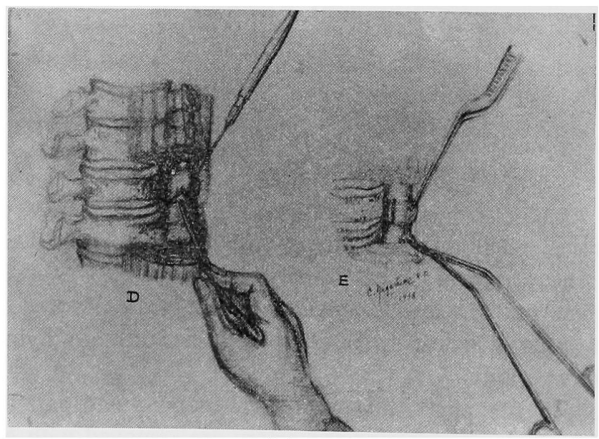

Fig. 2 